\title{
CPAP ohne Effekt auf kardiovaskuläres Risiko
}

Fragestellung: Ziel der vorliegenden Studie war es, den Effekt einer nächtlichen CPAP-Beatmung (continuous positive airway pressure) auf das Auftreten kardio- und zerebrovaskulärer Endpunkte bei Patienten mit koronarer Herzerkrankung (KHK) oder Schlaganfall und obstruktiver Schlafapnoe zu untersuchen.

Hintergrund: Große prospektive Beobachtungsstudien wie die „Sleep Heart Health Study“ oder die „Wisconsin Sleep Cohort Study“ zeigten, dass die obstruktive Schlafapnoe (OSA) das kardio- und zerebrovaskuläre Risiko erhöht $[1,2,3,4,5]$. Zwei nicht randomisierte Studien mit zusammen 2.767 Patienten $[4,5]$ suggerieren, dass der leitliniengerechte nächtliche Einsatz einer CPAP-Beatmung das kardiovaskuläre Risiko senken kann.

Patienten und Methodik: In die multizentrische SAVE-Studie wurden in Australien, China, Indien, Spanien, Brasilien und in den USA insgesamt 2.717 Patienten im Alter von 45 bis 75 Jahren mit einer moderat bis schwer ausgeprägten OSA, aber ohne relevante Tageschläfrigkeit eingeschlossen, die je etwa zur Hälfte eine manifeste KHK aufwiesen

McEvoy RD, Antic NA, Heeley E et al. CPAP for prevention of cardiovascular events in obstructive sleep apnea. N Engl J Med 2016; 375: $919-31$ oder einen ischämischen Schlaganfall erlitten hatten. Die Randomisierung erfolgte $1: 1$ in eine Gruppe mit CPAPTherapie und in eine Vergleichsgruppe ohne CPAP.
Der primäre Studienendpunkt umfasste kardiovaskulären Tod, Myokardinfarkt, Schlaganfall, instabile Angina pectoris, akute Herzinsuffizienz oder transitorische ischämische Attacke (TIA). Als sekundäre Endpunkte wurden unter anderem die Lebensqualität, die Stimmungslage und die Tagesschläfrigkeit untersucht.

Ergebnisse: Nach einem mittleren Follow-up von 3,7 Jahren hatten $17,0 \%$ der Patienten mit CPAP und 15,4\% der Patienten, die nicht mit CPAP behandelt wurden, den primären Endpunkt erreicht. Dies entsprach einer Hazard ratio (HR) von 1,10 (95\%-Konfidenzintervall [95\%-KI] 0,91-1,32) und war nicht signifikant $(\mathrm{p}=0,34)$. Auch für den Schlaganfall, der bei $5 \%$ aller Studienteilnehmer auftrat, fand sich kein signifikanter Unterschied in beiden Gruppen (HR 0,97; $95 \%$-KI 0,69-1,35). Hingegen führte die CPAP-Therapie zu einer signifikanten Reduktion der Tagesschläfrigkeit sowie zu einer signifikanten Verbesserung der subjektiven Lebensqualität und der Stimmungslage. Die durchschnittliche CPAP-Nutzungsdauer in der Interventionsgruppe betrug 3,3 Stunden pro Nacht bei einer mittleren Schlafzeit von sieben Stunden.

Schlussfolgerungen: Die Autoren folgern, dass die nächtliche CPAP-Therapie bei Patienten mit moderat bis schwer ausgeprägter OSA und bereits manifester KHK oder ischämischem Schlaganfall nicht zu einer Senkung des kardio- beziehungsweise zerebrovaskulären Risikos führt.

\section{- Kommentar von Farid Salih und Karl Georg Häusler, Berlin}

\section{Die Schlussfolgerungen nicht vorschnell in die Praxis übertragen}

In dieser bisher größten randomisierten Studie zur CPAP-Therapie bei Patienten mit OSA und KHK oder stattgehabtem Schlaganfall konnte kein sekundärpräventiver Effekt festgestellt werden, was den Ergebnissen von zwei nicht randomisierten Studien widerspricht $[4,5]$. Es ist zu kritisieren, dass die CPAP-Nutzungsdauer im Interventionsarm lediglich 3,3 Stunden pro Nacht und damit weniger als $50 \%$ der Gesamtschlafzeit betrug, was das (negative) Studienergebnis maßgeblich beeinflusst haben könnte. Allerdings ergab sich aus Patientensicht eine Verbesserung von Lebensqualität, Stimmungslage und Tagesschläfrigkeit unter der CPAP-Therapie. Leider wurden Patienten mit OSA und schweren nächtlichen $\mathrm{O}_{2}$-Entsättigungen sowie ausgeprägter Tagesschläfrigkeit (für die der Nutzen einer CPAP-Therapie wohl am größten sein dürfte) nicht in die SAVE-Studie eingeschlossen, möglicherweise um diesen Patienten eine CPAP-Therapie nicht vorzuenthalten. Die oben genannten Schlussfolgerungen der Autoren sollten somit nicht vorschnell in die klinische Praxis übertragen werden. Leider wurde weder eine Subgruppenanalyse zum möglichen Einfluss einer überdurchschnittlich hohen CPAP-Nutzungsdauer noch eine separate Analyse der Patienten publiziert, die bereits vor Einschluss in die SAVE-Studie einen ischämischen
Schlaganfall erlitten hatten. Inwieweit Unterschiede in der medizinischen Versorgung in einzelnen an der Rekrutierung beteiligten Ländern bestehen, die die Übertragbarkeit der Ergebnisse der SAVE-Studie beeinflussen, kann anhand der vorliegenden Daten nicht abschließend beantwortet werden.

\footnotetext{
Referenzen:

1. Arzt M et al. Am J Respir Crit Care Med 2005; 172: 1447 - 51

2. Redline S et al. Am J Respir Crit Care Med 2010; 182: 269- 77

3. Gottlieb DJ et al. Circulation 2010; 122: 352-60

4. Campos-Rodriguez F et al. Ann Intern Med 2012; 156: 115 - 22

5. Marin JM et al. Lancet 2005; 365: 1046-53
}

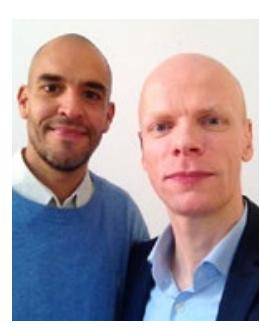

Dr. med. Farid Salih und PD Dr. med. Karl Georg Häusler, Berlin

Klinik und Hochschulambulanz für Neurologie, Charité - Universitätsmedizin Berlin. E-Mail: farid.salih@charite.de, georg.haeusler@charite.de 